\title{
Safety Profile and Effects of Pulsed Methylprednisolone on Vital Signs in Thyroid Eye Disease
}

\author{
Kai-Ling Yong, ${ }^{1,2}$ Chiaw Ling Chng, ${ }^{3,4}$ Hla Myint Htoon, \\ Lee Hooi Lim, ${ }^{1,2}$ and Lay Leng Seah ${ }^{1,2,5}$ \\ ${ }^{1}$ Singapore National Eye Centre, 11 Third Hospital Avenue, Singapore 168751 \\ ${ }^{2}$ Singapore Eye Research Institute, 11 Third Hospital Avenue, Singapore 168751 \\ ${ }^{3}$ Singapore General Hospital, Outram Road, Singapore 169608 \\ ${ }^{4}$ Duke-NUS Graduate Medical School, 8 College Road, Singapore 169857 \\ ${ }^{5}$ Yong Loo Lin School of Medicine, National University of Singapore, $1 E$ Kent Ridge Road, NUHS Tower Block, \\ Level 11, Singapore 119228 \\ Correspondence should be addressed to Kai-Ling Yong; insuria@yahoo.com
}

Received 12 September 2015; Revised 3 November 2015; Accepted 4 November 2015

Academic Editor: Franco Veglio

Copyright (c) 2015 Kai-Ling Yong et al. This is an open access article distributed under the Creative Commons Attribution License, which permits unrestricted use, distribution, and reproduction in any medium, provided the original work is properly cited.

Objective. To analyze changes in vital signs (heart rate (HR), systolic (SBP), and diastolic blood pressure (DBP)) during and after intravenous methylprednisolone (IVMP) and any other adverse effects. Methods. Retrospective review of charts of patients who received IVMP as treatment regime for thyroid eye disease. All subjects had vital signs charted during and after infusions. Results. This study included 38 subjects and a total of 242 infusions administered. IVMP resulted in a small but significant percentage drop in mean SBP at $30 \mathrm{~min}(p<0.001)$ and $60 \mathrm{~min}(p=0.03)$ but no difference at $90 \mathrm{~min}$. There was also small but significant percentage drop in mean DBP and HR (DBP: $p<0.001$ for $30 \mathrm{~min}, p=0.001$ for $60 \mathrm{~min}$, and $p=0.02$ for 90 min and HR: $p<0.001$ for $30 \mathrm{~min}, 60 \mathrm{~min}$, and $90 \mathrm{~min}$ ). There were no cumulative effects on change of blood pressure or HR. There were 6 episodes of bradycardia (2.5\%) and 12 episodes of moderate to severe hypertension (5\%). No significant cardiovascular or hepatic toxicity was found. Conclusion. IVMP is relatively safe and efficacious. IVMP demonstrated mild and noncumulative effects on vital signs. Severe hypertension may occur in susceptible individuals such as those with underlying hypertension and uncontrolled thyroid dysfunction, whereas bradycardia may be more likely in those on beta-blockers.

\section{Introduction}

Thyroid eye disease (TED) is an autoimmune process which involves abnormal proliferation and activation of orbital fibroblasts, increased glycosaminoglycans, and proinflammatory cytokine production. Cytokines can modulate the immune reaction in TED by increasing major histocompatibility complex class II, adhesion molecules, prostaglandin, and heat shock protein expression in the orbit $[1,2]$. Glucocorticoid therapy is effective in TED as it has anti-inflammatory and immunosuppressive actions, inhibits release of immune mediators such as cytokines, interferes with function of $\mathrm{T}$ and B lymphocytes, and decreases glycosaminoglycan production by orbital fibroblast $[3,4]$.
Pulsed intravenous methylprednisolone (IVMP) therapy is an established treatment of active moderate to severe TED and sight-threatening dysthyroid optic neuropathy [5]. IVMP therapy is also more efficacious and associated with fewer side effects than oral steroids $[6,7]$. The favourable response rates of pulsed IVMP are about $80 \%$, as compared with about $60 \%$ for oral steroids [8].

However, there are concerns regarding the safety profile of pulsed IVMP, especially on the cardiovascular system. There are many known adverse effects of pulsed IVMP therapy. Minor effects include flushing, mild hypertension, gastritis, weight gain, depression, hyperglycemia, insomnia, palpitations, sinusitis, and urinary tract infections [7, 9-12]. Major adverse events reported include cardiovascular events, acute 
liver damage, and even death [13-18]. There is no established consensus on the monitoring of patients during and after IVMP administration. Previous studies had reported IVMP resulting in hypertension in TED patients $[9,19]$ or bradycardia $[20,21]$, but none looked at the extent and pattern of blood pressure and heart rate fluctuations at various time points after the steroid infusions. In addition, literature detailing the risk factors for such fluctuations is scarce $[9,21]$.

The purpose of this study is to analyze the changes in vital signs (including heart rate (HR), systolic (SBP), and diastolic blood pressure (DBP)) during and after IVMP infusion ( $0 \mathrm{~min}, 30 \mathrm{~min}, 60 \mathrm{~min}$, and $90 \mathrm{~min}$ ), as well as changes in electrocardiogram (ECG), potassium levels, glucose levels, and any other adverse effects.

\section{Methods}

We performed a retrospective review of the charts of TED patients who underwent pulsed methylprednisolone therapy from 2004 to 2010 in Singapore National Eye Centre. Numerous different parenteral regimes for IVMP have been reported, with no clear consensus on the optimum dosage, dosing intervals, and duration of treatment [8, 22]. The dosing regimen used in our institution during the study period consisted of $1 \mathrm{~g}$ hourly per day over 3 days given in the outpatient setting $[11,23,24]$. The indications of IVMP were active TED or compressive optic neuropathy. The study was carried out in accordance with the tenets of World Medical Association's Declaration of Helsinki.

All subjects were examined for best correctable visual acuity (BCVA), colour vision, VISA scoring, and slit-lamp examination prior to pulsed methylprednisolone therapy. An inflammatory score of 4 or more on VISA was considered as active TED [25]. Baseline blood investigations including full blood count, liver function test, renal panel, glucose, hepatitis $\mathrm{B}$ and hepatitis C screening, and ECG were performed. Patients who had any symptoms or signs of infection, severe gastric disease, unstable cardiac disease, active liver disease, and poorly controlled diabetes mellitus or hypertension were excluded from IVMP treatment.

All subjects had their vital signs (HR, SBP, and DBP) charted before infusion ( $0 \mathrm{~min})$, at $30 \mathrm{~min}$ (during infusion) and $60 \mathrm{~min}$ (end of infusion), and after infusion (90 min). ECG and capillary glucose level were checked at $60 \mathrm{~min}$. Serum potassium was measured before and after each infusion for all subjects before discharge. All subjects had to complete a questionnaire at the end of infusion which documented any subjective complications experienced.

Subjects were reviewed after each cycle of 3 doses of IVMP for BCVA, colour vision, VISA score, and full slit-lamp examination. Full blood count, liver function test, and renal panel were performed. Subjects were reviewed at interval of 1 to 3 months after treatment depending on their condition and physician's discretion. Subjects may undergo repeated cycles of IVMP if the TED was considered active or patients developed signs of compressive optic neuropathy. Subjects may also be given adjuvant methotrexate or radiotherapy after IVMP treatments were completed.
TABLE 1: Characteristics of study population.

\begin{tabular}{lc}
\hline & Overall $(N=38)$ \\
\hline Mean age (SD), years & $48.81 \pm 8.8$ \\
Male (ratio) & $25(65.8 \%)$ \\
Mean number of cycles (SD) & $2.34 \pm 1.53$ \\
$\quad$ Range & $1-7$ \\
Smoking status & \\
Current smokers & $10(26.3 \%)$ \\
Nonsmokers & $24(63.2 \%)$ \\
Ex-smokers & $4(10.5 \%)$ \\
Preexisting comorbidities & \\
Heart disease & $3(7.9 \%)$ \\
Hyperlipidemia & $4(10.5 \%)$ \\
Stroke & $1(3.2 \%)$ \\
Diabetes & $7(18.4 \%)$ \\
Preexisting hepatitis (hepatitis B carrier) & $3(9.1 \%)$ \\
Indication for IVMP & \\
Active TED & $26(68.4 \%)$ \\
Compressive optic neuropathy & $12(31.6 \%)$ \\
Adjuvant therapy and subsequent surgery & \\
Methotrexate & $26(68.4 \%)$ \\
Radiotherapy & $10(26.3 \%)$ \\
Decompression surgery & $8(21.1 \%)$ \\
Mean duration of hyperthyroidism (SD), years & $3.56 \pm 5.76$ \\
Thyroid status & \\
Euthyroid & $9(23.7 \%)$ \\
Hyperthyroid & $22(57.9 \%)$ \\
Hypothyroid & $6(15.8 \%)$ \\
\hline
\end{tabular}

SD: standard deviation; IVMP: intravenous methylprednisolone; TED: thyroid eye disease.

Statistical analysis was performed with SPSS software (IBM Corp., released 2010, IBM SPSS Statistics for Windows, Version 19.0, Armonk, NY). Parametric data was analyzed using paired $t$-test. A linear mixed model was utilized to analyze and account for the cumulative effect of the multiple doses of each cycle of methylprednisolone. Three candidate covariance structures were checked for minimizing the mean squared error of predictions, first-order autoregressive $[\mathrm{AR}(1)]$, compound symmetry (CS), and unstructured (UN), to estimate the fixed effects and we have selected the structure with the smallest Akaike's Information Criterion (AIC) and Bayesian's Information Criterion (BIC). Linear mixed models were fitted and compared using restricted maximum likelihood methods. A statistical significance level of 0.05 was used for this study.

\section{Results}

The study included 38 subjects who underwent a total of 242 infusions of IVMP (Table 1). The mean age (standard deviation, SD) was $48.4 \pm 8.8$ years and $65.8 \%$ were male. The subjects underwent a mean (SD) of $2.3 \pm 1.5$ cycles of IVMP (range: 1 to 7 cycles). The main indication of therapy was active TED (68.4\%). Twenty-six subjects required 


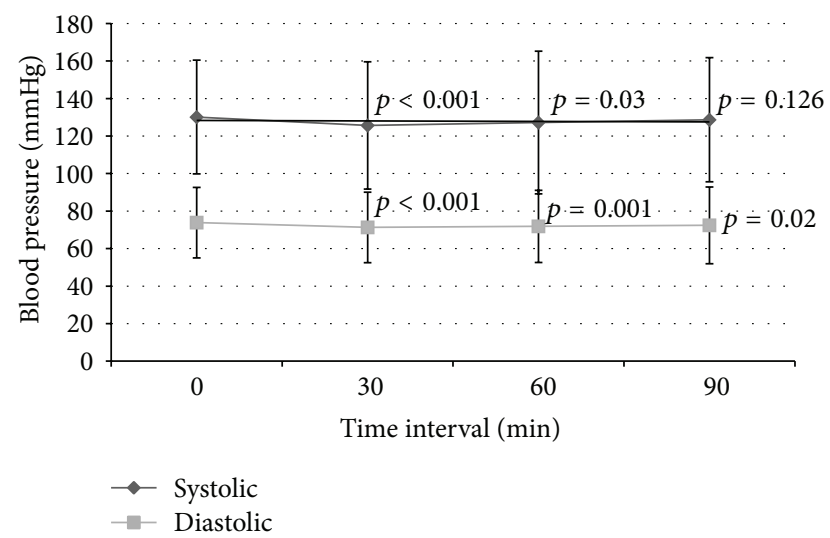

FIGURE 1: Systolic and diastolic blood pressure over time. Significance value pairwise comparison for SBP (paired $t$-test): $p<0.001$ for $0 \mathrm{~min}$ versus $30 \mathrm{~min}, p=0.03$ for $0 \mathrm{~min}$ versus $60 \mathrm{~min}$, and $p=0.126$ for $0 \mathrm{~min}$ versus $90 \mathrm{~min}$. Significance value pairwise comparison for DBP (paired $t$-test): $p<0.001$ for 0 min versus $30 \mathrm{~min}, p=0.001$ for $0 \mathrm{~min}$ versus $60 \mathrm{~min}$, and $p=0.02$ for $0 \mathrm{~min}$ versus $90 \mathrm{~min}$.

adjuvant therapy of methotrexate, and 10 subjects required radiotherapy. Eight subjects went for subsequent decompression surgery. More than half (57.9\%) of the subjects showed hyperthyroidism (defined as raised free thyroxine with a suppressed thyroid stimulating hormone) on thyroid function test at baseline.

There was a small but statistically significant percentage drop in mean (SD) SBP at $30 \mathrm{~min}$ and $60 \mathrm{~min}$ compared to baseline SBP $(-3.31 \pm 9.91 \%, p<0.001$, and $-2.25 \pm 12.18 \%$, $p=0.03$, resp.) but no significant difference after stopping infusion at $90 \mathrm{~min}(-0.67 \pm 11 \%, p=0.126)$. There was also small but statistically significant drop in mean percentage change DBP at $30 \mathrm{~min}, 60 \mathrm{~min}$, and $90 \mathrm{~min}$ compared to baseline DBP $(-2.92 \pm 10.6 \%, p<0.001,-2.1 \pm 11.82 \%$, $p=0.001$, and $-1.34 \pm 12.63 \%, p=0.02$, resp.) (Figure 1 ). The IVMP resulted in a significant decrease in mean percentage change $\mathrm{HR}(p<0.001)$ from $0 \mathrm{~min}$ to $30 \mathrm{~min}(-5.73 \pm$ $10.14 \%), 60 \mathrm{~min}(-5.58 \pm 11.36 \%)$, and $90 \mathrm{~min}(-5.92 \pm 12.12 \%)$ (Figure 2). After comparing the SBP, DBP, and HR between the 3 doses of IVMP, there were no statistically significant cumulative effects of methylprednisolone on percentage change of SBP, DBP, and HR between doses 1, 2, and 3 within each IVMP cycle.

There was a significant decrease $(p=0.045)$ in mean (SD) potassium levels from $4.14 \pm 0.36 \mathrm{mmol} / \mathrm{L}$ to $4.08 \pm$ $0.37 \mathrm{mmol} / \mathrm{L}$, but all were within normal clinical range. There was no significant cumulative effect on potassium levels between each dose of the cycle $(p=0.837)$. The mean (SD) random capillary glucose level was significantly increased from $7.76 \pm 3.28 \mathrm{mmol} / \mathrm{g}$ to $11.87 \pm 5.87 \mathrm{mmol} / \mathrm{g}$ after infusion $(p<0.001)$. There was also no significant cumulative effect on glucose levels between each dose $(p=0.563)$. There were 4 patients $(10.5 \%)$ who required subcutaneous insulin therapy to correct the hyperglycemia, and all these subjects had preexisting type 2 diabetes mellitus.

Overall, there was improvement in both BCVA and colour vision after treatment after each cycle of 3 doses of $1 \mathrm{~g}$

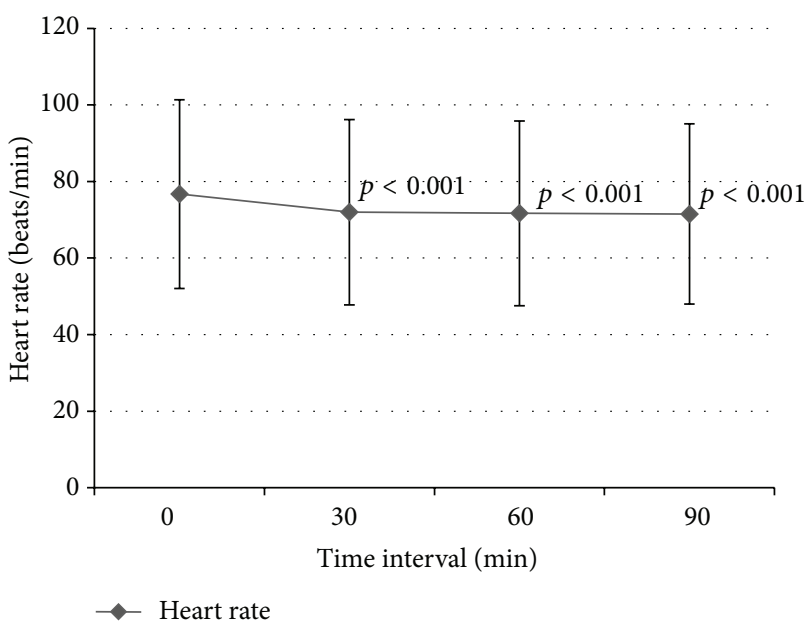

FIGURE 2: Heart rate over time. Significance value pairwise comparison for heart rate (paired $t$-test): $p<0.001$ for 0 min versus $30 \mathrm{~min}$, $p<0.001$ for $0 \mathrm{~min}$ versus $60 \mathrm{~min}$, and $p<0.001$ for $0 \mathrm{~min}$ versus $90 \mathrm{~min}$.

TABLE 2: Outcomes.

\begin{tabular}{|c|c|c|}
\hline & Pre-infusion & Post-infusion \\
\hline Mean VA, right $(\mathrm{SD}) / \log \mathrm{Mar}^{\mathrm{a}}$ & $0.28(0.29)$ & $0.16(0.26)$ \\
\hline Mean VA, left (SD)/log Mara & $0.27(0.28)$ & $0.17(0.26)$ \\
\hline Mean colours, right $(\mathrm{SD})^{\mathrm{a}, \mathrm{b}}$ & $10.5(3.6)$ & $13.4(5.4)$ \\
\hline Mean colours, left $(S D)^{a, b}$ & $13.1(4.2)$ & $13.9(3.9)$ \\
\hline Mean VISA inflammatory score (SD) & $4.29(1.14)$ & $1.92(1.12)$ \\
\hline
\end{tabular}

VA: visual acuity; SD: standard deviation

${ }^{\mathrm{a}}$ Only those with compressive optic neuropathy were analyzed.

${ }^{\mathrm{b}}$ Colour vision was assessed using 15 plates of Ishihara colour vision test.

IVMP, although it was not statistically significant $(p=0.348$ and $p=0.685$ for BCVA right and left eye and $p=0.459$ and $p=0.641$ for colour vision right and left eye, resp.). However, if only the subjects with compressive optic neuropathy were analyzed, there was a significant improvement in both visual acuity and colour vision (Table 2 ). There was also significant improvement $(p<0.001)$ of VISA inflammatory score from mean score $(\mathrm{SD})$ of $4.29 \pm 1.14$ before treatment to $1.92 \pm 1.12$ after treatment.

Examining the overall adverse effects of IVMP, there were $6(2.5 \%)$ episodes of bradycardia (HR less than $50 \mathrm{bpm}), 8$ (3.3\%) episodes of tachycardia (HR more than $100 \mathrm{bpm})$, and $12(5 \%)$ episodes of moderate to severe hypertension or stage 2 hypertension (SBP $>160 \mathrm{mmHg}$ or DBP $>100 \mathrm{mmHg}$ ) [26]. These 12 episodes occurred in 4 subjects; one of them was hyperthyroid with no underlying hypertension and two were hypothyroid and hypertensive while one was euthyroid with underlying hypertension. Three subjects (1.2\%) had severe hypertension (as defined by SBP $\geq 180 \mathrm{mmHg}$ or DBP $\geq$ $110 \mathrm{mmHg}$ ) (Table 3). All these subjects had documented normal blood pressure before initiation of IVMP. One patient developed urticarial rash and the pulsed methylprednisolone was stopped immediately and hence did not complete the cycle of 3 doses of therapy. The patient's vital signs remained stable. Two patients (5.3\%) had mildly deranged 
TABLE 3: Side effects (out of 242 infusions).

\begin{tabular}{lcc}
\hline Side effects & $N$ & $\%$ \\
\hline Bradycardia $<50 \mathrm{bpm}$ & 6 & 2.5 \\
Hypertension SBP $>160$ mmHg and/or DBP $>100 \mathrm{mmHg}$ & 12 & 5 \\
Urticarial Rash-IVMP stopped $^{\mathrm{a}}$ & 1 & 0.4 \\
Nonspecific rashes or flushing $^{\mathrm{a}}$ & 5 & 2.1 \\
Palpitations $^{\mathrm{a}}$ & 2 & 0.8 \\
Shortness of breath $^{\mathrm{a}}$ & 2 & 0.8 \\
Chest tightness $^{\mathrm{a}}$ & 1 & 0.4 \\
Headache $^{\mathrm{a}}$ & 6 & 2.5 \\
Insomnia $^{\mathrm{a}}$ & 2 & 0.8 \\
Fever $^{\mathrm{a}}$ & 1 & 0.4 \\
Gastritis $^{\mathrm{a}}$ & 1 & 0.4 \\
Changes in LFT $^{\mathrm{b}}$ & 0 & 0 \\
Changes in ECG & 0 & 0 \\
\hline
\end{tabular}

SBP: systolic blood pressure, DBP: diastolic blood pressure, IVMP: intravenous methylprednisolone, LFT: liver function test, and ECG: electrocardiogram.

${ }^{a}$ Documented from questionnaire after end of each infusion at $90 \mathrm{~min}$.

${ }^{b}$ Defined as above, 3 times of upper limit of normal.

liver enzymes, which all reverted to normal by 1 year after the administration of IVMP. No new arrhythmias or ischemic events were noted on ECG monitoring for all subjects. Two subjects complained of breathlessness and 1 had chest tightness, with no accompanying vital sign or ECG changes. These symptoms did not recur during the subsequent doses of IVMP in the two subjects.

\section{Discussion}

Pulsed methylprednisolone infusion for treatment of active TED has been shown to be relatively safe and effective. IVMP therapy is established to be effective in improving inflammation in active TED, more efficacious than oral steroids with less adverse events $[8,27]$. Consistent with the current literature, our study showed that these patients had improved inflammatory score and those with compressive optic neuropathy had improvement in visual acuity.

The mechanisms by which steroids lead to hypertension may be multifactorial. Steroids result in sodium retention and volume expansion [28] and act on nitric oxide synthase pathway [29]. Steroids may also mediate vascular vasoconstrictor sensitivity to catecholamines and other vasoconstrictor hormones systems [30,31]. Our results showed that IVMP had a general trend in lowering the SBP and DBP; however the mean change was small and may not be clinically significant. Although the effects of IVMP on BP were not cumulative in our study, we found 12 (5\%) episodes of moderate to severe hypertension (SBP $\geq 160 \mathrm{mmHg}$ or DBP $\geq 100 \mathrm{mmHg}$ ), and three subjects had SBP $180 \mathrm{mmHg}$ or more at the end of infusion. All these patients' blood pressure returned to normal at 90 minutes and remained asymptomatic. Subsequent administration of IVMP did not cause such dramatic changes in blood pressure. Nonetheless, IVMP should be used with caution in those with poorly controlled hypertension since sudden fluctuation in blood pressure may still exert stress on the cardiac system and result in pulmonary oedema and acute heart failure [13].

There were three patients with thyroid dysfunction at baseline who developed moderate to severe hypertension during IVMP infusion. Both hyperthyroidism and hypothyroidism are associated with increased blood pressure; hypothyroidism is a recognised cause of secondary hypertension [32], and hyperthyroidism results in higher risk of elevated SBP, widened pulse pressure, sinus tachycardia, and atrial fibrillation and may even lead to heart failure [33-35].

IVMP administration has been associated with cardiac arrhythmias in some studies [20,36]. Although IVMP resulted in decrease in HR that remained low at 90 min after IVMP infusion in our study, there was no cumulative effect on the subsequent dose the next day. All the subjects who developed bradycardia in our study were on beta-blockers, while the subjects who developed tachycardia were hyperthyroid and were already tachycardic at baseline. None of the patients developed atrial fibrillation. Our study suggests that patients on beta-blockers were more susceptible to developing bradycardia. Bradycardia after steroid administration had been reported, but the mechanisms were not well studied $[20,21]$. One explanation for the occurrence of reversible bradycardia is by the pharmacokinetics of steroid operating through the hypothalamic-pituitary axis suppression [37].

IVMP may also cause hyperglycemia, especially in diabetic patients and transient deranged liver enzymes; thus careful screening of patient's glucose levels and liver function should be performed prior to IVMP administration $[10,38]$. In our study, the four subjects who required insulin therapy for correction of hyperglycemia were all diabetics. Similarly in a large study by Feldman-Billard et al. [10], the authors found that methylprednisolone therapy resulted in raised glucose levels, but the effects were different in nondiabetics and diabetics. In nondiabetics, the hyperglycemia was maximum after first pulse and returned to baseline with subsequent pulses, but diabetic patients had cumulative effects of the hyperglycemia with repeated pulses, and a quarter of the diabetic patients required insulin. Our study showed that four out of seven diabetic patients required insulin at some point during their cycles but did not show any overall cumulative effects of glucose with repeated pulses. However, our study did not analyze the cumulative effects on diabetics alone due to the small numbers. Although the hyperglycemic effects may be transient and correctable, acute hyperglycemia is an independent risk factor of cardiovascular events and should be prevented [39, 40].

Hypertension and hyperglycemia may also result in pathophysiological changes in the retina, such as arteriolar narrowing, retinal haemorrhages, microaneurysms, exudates, and optic disc swelling [41, 42]. Although the hypertensive effects of methylprednisolone may be transient, the acute increase of blood pressure may lead to vascular necrosis, resulting in necrosis of smooth muscle and endothelial cells [43]. Acute retinopathy changes associated with hypertension may be generally reversible, but potential worsening of diabetes control induced by repeated pulsed high dose methylprednisolone therapy may exert irreversible damage to end target organs through the "metabolic memory" [43]. 
Marinó et al. estimated acute liver damage in $0.8 \%$ of TED patients who underwent IVMP [15]. They postulated that IVMP caused liver damage by either direct damage on liver cells, precipitation of virus-induced hepatitis, or reactivation of immune system after IVMP-induced immune suppression resulting in autoimmune hepatitis. The damage may be dose dependent [44]. Our subjects underwent a large range of IVMP courses (one to seven cycles), and seven patients had cumulative doses of more than $9 \mathrm{~g}$. Of note, six of these subjects were treated between 2004 and 2007, which were before the recommendation that IVMP should not exceed cumulative dose of $8 \mathrm{~g}$ [17]. One subject had seven cycles administered in parts over three years. None of our patients developed liver failure, and the two subjects who had mild increase in liver enzymes (less than three times of the upper reference limit) had less than $8 \mathrm{~g}$ administered. Their liver function tests returned to normal within 1 year after the IVMP pulses were stopped. In our study, one subject developed urticarial rash near the end of the 60-minute infusion. Anaphylactic shock had been reported in IVMP administration $[45,46]$; hence it is recommended that the drug be infused slowly over $60 \mathrm{~min}$.

Despite demonstrating a decrease in potassium levels after IVMP infusion, all the subjects' potassium levels were within normal clinical range. This may suggest that in relatively well patients with normal potassium at baseline, there is no need to recheck their potassium levels after IVMP administration. The caveat is that these patients should not have any underlying medical conditions that may result in them having electrolyte abnormalities, such as renal failure or use of medications such as diuretics.

The main strengths of our study were the large number of 242 infusions analyzed and recorded patients who had multiple infusions administered with the long follow-up duration. The main limitations were the small number of study subjects and the large variance of dosage administered (cumulative dose: $1 \mathrm{~g}$ to $21 \mathrm{~g}$ ) instead of a fixed cumulative dosage administered. However, such variation is expected in the management of patients with active TED where response to IVMP may be variable and other forms of second-line treatment may be required at different time points during the course of treatment.

\section{Conclusion}

IVMP is a relatively safe and efficacious therapy for active moderate to severe TED and optic neuropathy. Overall, IVMP administration resulted in mild and noncumulative effects on heart rate and blood pressure. However, patients with poorly controlled hypertension, diabetes, or active liver disease should have their underlying conditions optimised before undergoing pulsed methylprednisolone therapy. Severe hypertension may occur during IVMP treatment in susceptible individuals such as those with underlying hypertension and uncontrolled thyroid dysfunction, whereas bradycardia may be more likely in those on beta-blockers. Patients whose potassium levels are normal at baseline and are not predisposed to hypokalemia from drugs or medical conditions may not require close monitoring of their potassium levels after every dose.

\section{Conflict of Interests}

The authors declare that there is no conflict of interests regarding the publication of this paper.

\section{References}

[1] S. J. C. Shan and R. S. Douglas, "The pathophysiology of thyroid eye disease," Journal of Neuro-Ophthalmology, vol. 34, no. 2, pp. 177-185, 2014.

[2] R. A. Ajjan and A. P. Weetman, "New understanding of the role of cytokines in the pathogenesis of Graves' ophthalmopathy," Journal of Endocrinological Investigation, vol. 27, no. 3, pp. 237245, 2004.

[3] L. Bartalena, C. Marocci, F. Bogazzi, G. Bruno-Bossio, and A. Pinchera, "Glucocorticoid therapy of Graves' ophthalmopathy," Experimental and Clinical Endocrinology \& Diabetes, vol. 97, no. 2-3, pp. 320-327, 1991.

[4] T. J. Smith and N. Hoa, "Immunoglobulins from patients with Graves' disease induce hyaluronan synthesis in their orbital fibroblasts through the self-antigen, insulin-like growth factor-I receptor," Journal of Clinical Endocrinology and Metabolism, vol. 89, no. 10, pp. 5076-5080, 2004.

[5] L. Bartalena, L. Baldeschi, A. Dickinson et al., "Consensus statement of the European Group on Graves' orbitopathy (EUGOGO) on management of GO," European Journal of Endocrinology, vol. 158, no. 3, pp. 273-285, 2008.

[6] P. E. Macchia, M. Bagattini, G. Lupoli, M. Vitale, G. Vitale, and G. Fenzi, "High-dose intravenous corticosteroid therapy for Graves' ophthalmopathy," Journal of Endocrinological Investigation, vol. 24, no. 3, pp. 152-158, 2001.

[7] G. J. Kahaly, S. Pitz, G. Hommel, and M. Dittmar, "Randomized, single blind trial of intravenous versus oral steroid monotherapy in Graves' orbitopathy," Journal of Clinical Endocrinology and Metabolism, vol. 90, no. 9, pp. 5234-5240, 2005.

[8] S. Zang, K. A. Ponto, and G. J. Kahaly, "Intravenous glucocorticoids for Graves' orbitopathy: efficacy and morbidity," The Journal of Clinical Endocrinology \& Metabolism, vol. 96, no. 2, pp. 320-332, 2011.

[9] L. Bartalena, G. E. Krassas, W. Wiersinga et al., "Efficacy and safety of three different cumulative doses of intravenous methylprednisolone for moderate to severe and active Graves' orbitopathy," Journal of Clinical Endocrinology and Metabolism, vol. 97, no. 12, pp. 4454-4463, 2012.

[10] S. Feldman-Billard, B. Lissak, R. Kassaei, R. Benrabah, and E. Héron, "Short-term tolerance of pulse methylprednisolone therapy in patients with diabetes mellitus," Ophthalmology, vol. 112, no. 3, pp. 511-515, 2005.

[11] Y. Hiromatsu, K. Tanaka, M. Sato et al., "Intravenous methylprednisolone pulse therapy for Graves' ophthalmopathy," Endocrine Journal, vol. 40, no. 1, pp. 63-72, 1993.

[12] A. De Bellis, A. Bizzarro, M. Conte et al., "Relationship between longitudinal behaviour of some markers of eye autoimmunity and changes in ocular findings in patients with Graves' ophthalmopathy receiving corticosteroid therapy," Clinical Endocrinology, vol. 59, no. 3, pp. 388-395, 2003.

[13] A. Gursoy, M. Cesur, M. F. Erdogan, D. Çorapcioglu, and N. Kamel, "New-onset acute heart failure after intravenous 
glucocorticoid pulse therapy in a patient with Graves' ophthalmopathy," Endocrine, vol. 29, no. 3, pp. 513-516, 2006.

[14] M. E. Lendorf, Å. K. Rasmussen, H. C. Fledelius, and U. FeldtRasmussen, "Cardiovascular and cerebrovascular events in temporal relationship to intravenous glucocorticoid pulse therapy in patients with severe endocrine ophthalmopathy," Thyroid, vol. 19, no. 12, pp. 1431-1432, 2009.

[15] M. Marinó, E. Morabito, M. R. Brunetto, L. Bartalena, A. Pinchera, and C. Marocci, "Acute and severe liver damage associated with intravenous glucocorticoid pulse therapy in patients with Graves' ophthalmopathy," Thyroid, vol. 14, no. 5, pp. 403406, 2004.

[16] M. Weissel and W. Hauff, "Fatal liver failure after high-dose glucocorticoid pulse therapy in a patient with severe thyroid eye disease," Thyroid, vol. 10, no. 6, article 521, 2000.

[17] C. Marcocci, T. Watt, M. A. Altea et al., "Fatal and non-fatal adverse events of glucocorticoid therapy for Graves' orbitopathy: a questionnaire survey among members of the European Thyroid Association," European Journal of Endocrinology, vol. 166, no. 2, pp. 247-253, 2012.

[18] H. Eguchi, J. Tani, S. Hirao et al., "Liver dysfunction associated with intravenous methylprednisolone pulse therapy in patients with graves' orbitopathy," International Journal of Endocrinology, vol. 2015, Article ID 835979, 5 pages, 2015.

[19] R. J. van Geest, I. V. Sasim, H. P. F. Koppeschaar et al., "Methylprednisolone pulse therapy for patients with moderately severe Graves' orbitopathy: a prospective, randomized, placebocontrolled study," European Journal of Endocrinology, vol. 158, no. 2, pp. 229-237, 2008.

[20] A. Vasheghani-Farahani, M. A. Sahraian, L. Darabi, A. Aghsaie, and A. Minagar, "Incidence of various cardiac arrhythmias and conduction disturbances due to high dose intravenous methylprednisolone in patients with multiple sclerosis," Journal of the Neurological Sciences, vol. 309, no. 1-2, pp. 75-78, 2011.

[21] J. D. Akikusa, B. M. Feldman, G. J. Gross, E. D. Silverman, and R. Schneider, "Sinus bradycardia after intravenous pulse methylprednisolone," Pediatrics, vol. 119, no. 3, pp. e778-e782, 2007.

[22] D. H. Verity and G. E. Rose, "Acute thyroid eye disease (TED): principles of medical and surgical management," Eye, vol. 27, no. 3, pp. 308-319, 2013.

[23] K. Ohtsuka, A. Sato, S. Kawaguchi, M. Hashimoto, and Y. Suzuki, "Effect of steroid pulse therapy with and without orbital radiotherapy on Graves' ophthalmopathy," American Journal of Ophthalmology, vol. 135, no. 3, pp. 285-290, 2003.

[24] T. Tagami, K. Tanaka, H. Sugawa et al., "High-dose intravenous steroid pulse therapy in thyroid-associated ophthalmopathy," Endocrine Journal, vol. 43, no. 6, pp. 689-699, 1996.

[25] P. J. Dolman and J. Rootman, "VISA classification for graves orbitopathy," Ophthalmic Plastic and Reconstructive Surgery, vol. 22, no. 5, pp. 319-324, 2006.

[26] A. V. Chobanian, G. L. Bakris, H. R. Black et al., "Seventh report of the joint national committee on prevention, detection, evaluation, and treatment of high blood pressure," Hypertension, vol. 42, no. 6, pp. 1206-1252, 2003.

[27] H. Stiebel-Kalish, E. Robenshtok, M. Hasanreisoglu, D. Ezrachi, I. Shimon, and L. Leibovici, "Treatment modalities for Graves' ophthalmopathy: systematic review and metaanalysis," The Journal of Clinical Endocrinology \& Metabolism, vol. 94, no. 8, pp. 2708-2716, 2009.
[28] J. J. Kelly, G. Mangos, P. M. Williamson, and J. A. Whitworth, "Cortisol and hypertension," Clinical and Experimental Pharmacology and Physiology, vol. 25, pp. S51-S56, 1998.

[29] F. J. Frey, A. Odermatt, and B. M. Frey, "Glucocorticoid-mediated mineralocorticoid receptor activation and hypertension," Current Opinion in Nephrology and Hypertension, vol. 13, no. 4, pp. 451-458, 2004.

[30] B. R. Walker, A. A. Connacher, D. J. Webb, and C. R. W. Edwards, "Glucocorticoids and blood pressure: a role for the cortisol/cortisone shuttle in the control of vascular tone in man," Clinical Science, vol. 83, no. 2, pp. 171-178, 1992.

[31] M. E. Ullian, "The role of corticosteroids in the regulation of vascular tone," Cardiovascular Research, vol. 41, no. 1, pp. 55-64, 1999.

[32] S. Stabouli, S. Papakatsika, and V. Kotsis, "Hypothyroidism and hypertension," Expert Review of Cardiovascular Therapy, vol. 8, no. 11, pp. 1559-1565, 2010.

[33] I. Klein and K. Ojamaa, "Thyroid hormone and the cardiovascular system," The New England Journal of Medicine, vol. 344, no. 7, pp. 501-509, 2001.

[34] C. T. Sawin, "Subclinical hyperthyroidism and atrial fibrillation," Thyroid, vol. 12, no. 6, pp. 501-503, 2002.

[35] P. Iglesias, M. Acosta, R. Sánchez, M. J. Fernández-Reyes, C. Mon, and J. J. Díez, "Ambulatory blood pressure monitoring in patients with hyperthyroidism before and after control of thyroid function," Clinical Endocrinology, vol. 63, no. 1, pp. 6672, 2005.

[36] A. E. McLuckie and R. W. Savage, "Atrial fibrillation following pulse methylprednisolone therapy in an adult," Chest, vol. 104, no. 2, pp. 622-623, 1993.

[37] P. J. Pentikäinen, "Pharmacological aspects of corticosteroid pulse therapy," Scandinavian Journal of Rheumatology, vol. 13, no. 54, pp. 6-9, 1984.

[38] C. Marcocci and M. Marinò, "Treatment of mild, moderate-tosevere and very severe Graves' orbitopathy," Best Practice and Research: Clinical Endocrinology and Metabolism, vol. 26, no. 3, pp. 325-337, 2012.

[39] H. Su, X. Sun, H. Ma et al., "Acute hyperglycemia exacerbates myocardial ischemia/reperfusion injury and blunts cardioprotective effect of GIK," The American Journal of PhysiologyEndocrinology and Metabolism, vol. 293, no. 3, pp. E629-E635, 2007.

[40] Z. Yang, V. E. Laubach, B. A. French, and I. L. Kron, "Acute hyperglycemia enhances oxidative stress and exacerbates myocardial infarction by activating nicotinamide adenine dinucleotide phosphate oxidase during reperfusion," Journal of Thoracic and Cardiovascular Surgery, vol. 137, no. 3, pp. 723-729, 2009.

[41] R. Klein, B. E. K. Klein, S. E. Moss, and W. Tasman, "The relation of systemic hypertension to changes in the retinal vasculature: the Beaver Dam Eye Study," Transactions of the American Ophthalmological Society, vol. 95, pp. 329-350, 1997.

[42] R. Klein, B. E. K. Klein, S. E. Moss, M. D. Davis, and D. L. DeMets, "The Wisconsin Epidemiologic study of diabetic retinopathy. III. Prevalence and risk of diabetic retinopathy when age at diagnosis is 30 or more years," Archives of Ophthalmology, vol. 102, no. 4, pp. 527-532, 1984.

[43] A. Grosso, N. Cheung, F. Veglio, and T. Y. Wong, "Similarities and differences in early retinal phenotypes in hypertension and diabetes," Journal of Hypertension, vol. 29, no. 9, pp. 1667-1675, 2011. 
[44] R. Le Moli, L. Baldeschi, P. Saeed, N. Regensburg, M. P. Mourits, and W. M. Wiersinga, "Determinants of liver damage associated with intravenous methylprednisolone pulse therapy in Graves' ophthalmopathy," Thyroid, vol. 17, no. 4, pp. 357-362, 2007.

[45] C. A. Deruaz, F. Spertini, F. Souza Lima, R. A. Du Pasquier, and M. Schluep, "Anaphylactic reaction to methylprednisolone in multiple sclerosis: a practical approach to alternative corticosteroids," Multiple Sclerosis, vol. 13, no. 4, pp. 559-560, 2007.

[46] S. Schonwald, "Methylprednisolone anaphylaxis," The American Journal of Emergency Medicine, vol. 17, no. 6, pp. 583-585, 1999. 


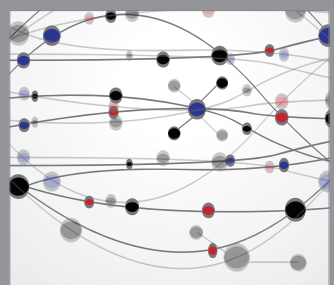

The Scientific World Journal
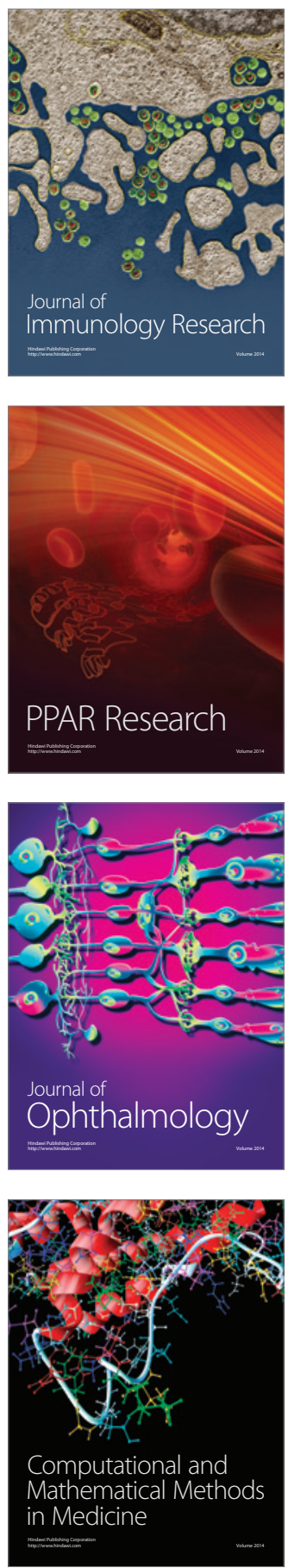

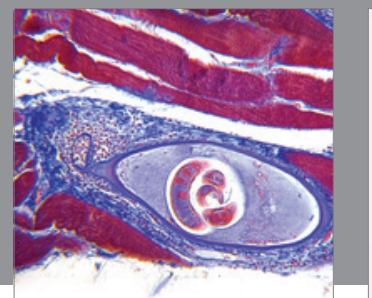

Gastroenterology

Research and Practice
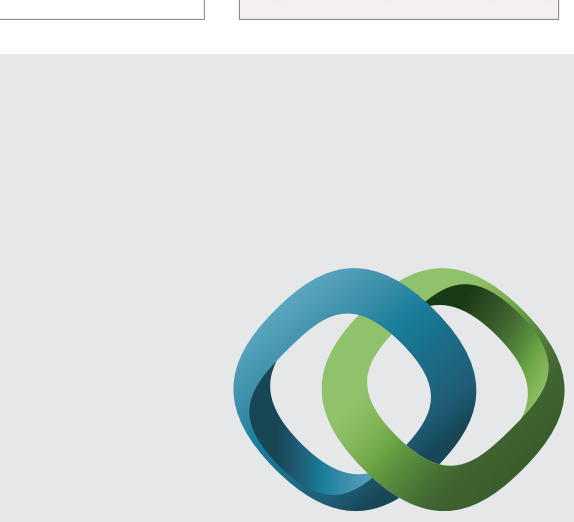

\section{Hindawi}

Submit your manuscripts at

http://www.hindawi.com
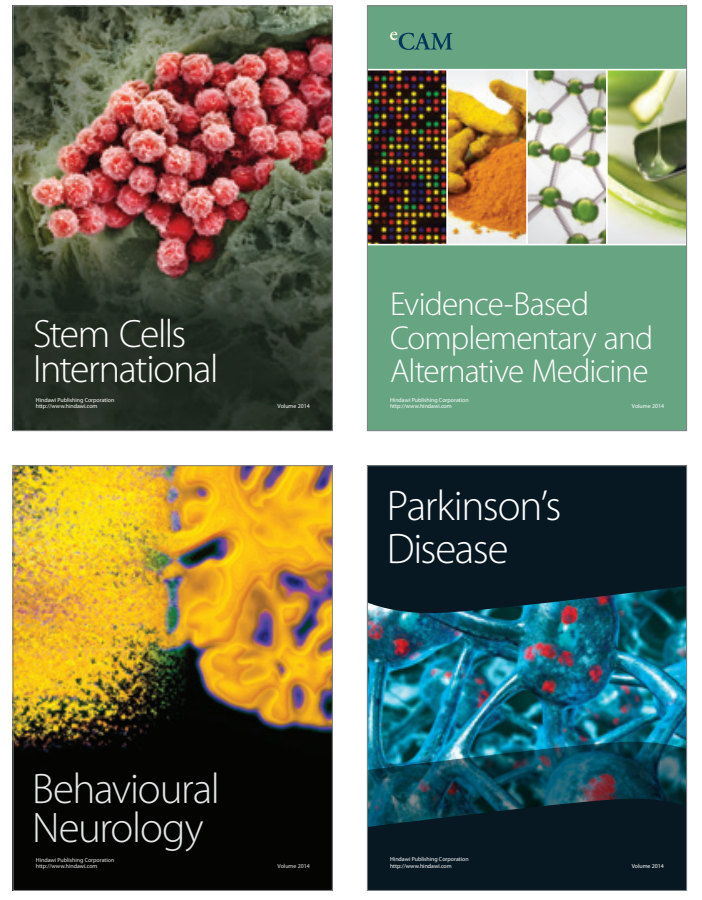
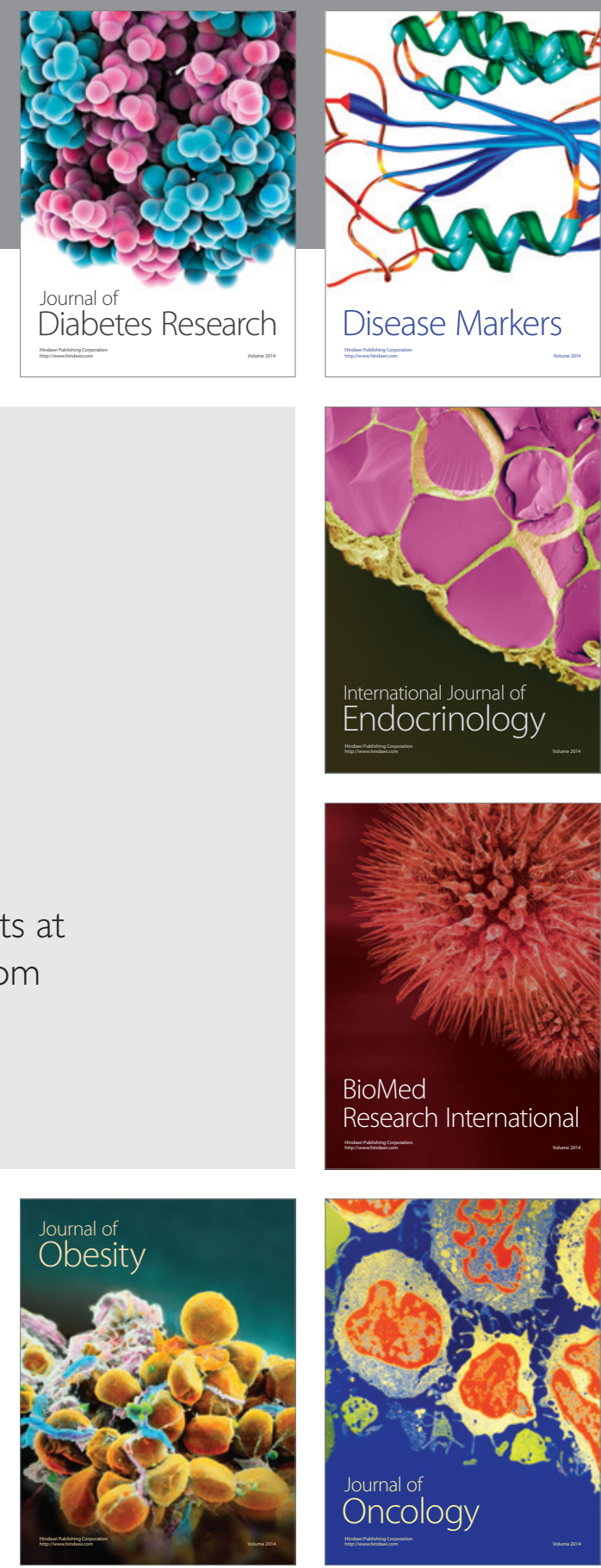

Disease Markers
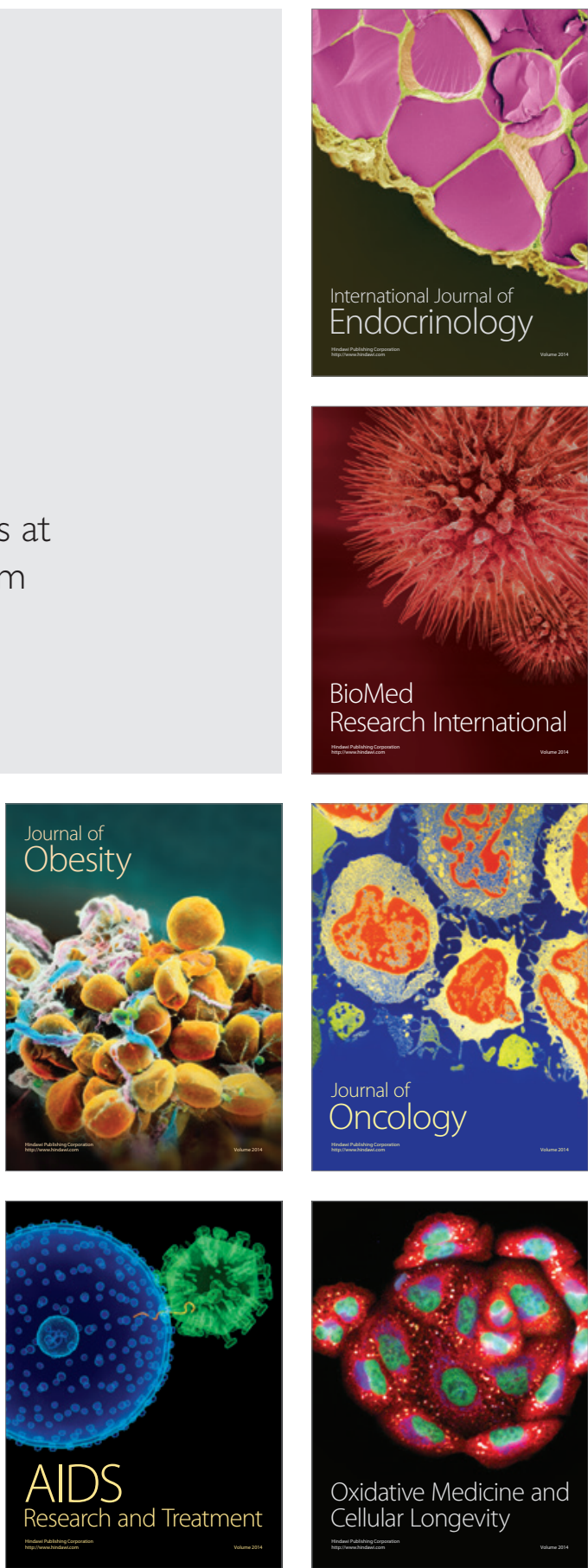